\title{
Immune profiling of Alzheimer patients
}

\author{
Mariavaleria Pellicanò a,*, Anis Larbi ${ }^{\text {b }}$, David Goldeck a , Giuseppina Colonna-Romano ${ }^{\text {, }}$, Silvio Buffa ${ }^{\text {, }}$, \\ Matteo Bulati ${ }^{\mathrm{c}}$, Graziella Rubino ${ }^{\mathrm{a}}$, Francesco Iemolo ${ }^{\mathrm{d}}$, Giuseppina Candore ${ }^{\mathrm{c}}$, Calogero Caruso ${ }^{\mathrm{C}}$, \\ Evelyna Derhovanessian ${ }^{a}$, Graham Pawelec ${ }^{a}$

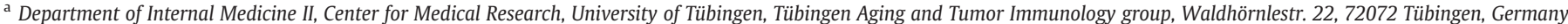 \\ b Singapore Immunology Network (SIgN), Immunos Building, Agency for Science, Technology and Research (A*STAR), Singapore \\ c Immunosenescence Unit, Department of Pathobiology and Medical and Forensic Biotechnologies, University of Palermo, Corso Tukory 211, 90134, Palermo, Italy \\ d U.O. di Neurologia, Ospedale di Vittoria, ASL7-Ragusa and Clinica Neurologica Università di Catania, Catania, Italy
}

\section{A R T I C L E I N F O}

\section{Article history}

Received 12 September 2011

Received in revised form 28 October 2011

Accepted 3 November 2011

\section{Keywords:}

Alzheimer's disease

T cells

Aß42

Immunosenescence

\begin{abstract}
A B S T R A C T
Alzheimer's disease $(A D)$ is characterized by extracellular senile plaques in the brain, containing amyloid- $\beta$ peptide $(A B)$. We identify immunological differences between $A D$ patients and age-matched controls greater than those related to age itself. The biggest differences were in the $\mathrm{CD} 4+$ rather than the $\mathrm{CD} 8+\mathrm{T}$ cell compartment resulting in lower proportions of naïve cells, more late-differentiated cells and higher percentages of activated CD4 + CD25 + T cells without a Treg phenotype in $\mathrm{AD}$ patients. Changes to $\mathrm{CD} 4+$ cells might be the result of chronic stimulation by $\mathrm{A} \beta$ present in the blood. These findings have implications for diagnosis and understanding the aetiology of the disease.
\end{abstract}

(c) 2011 Elsevier B.V. All rights reserved.

\section{Introduction}

Alzheimer's disease (AD) is an age-related neurological disorder that leads to progressive dementia. AD is histopathologically characterized by extracellular amyloid plaques, formed by amyloid beta peptide $(A \beta)$, and by intracellular neurofibrillary tangles. Deposits of highly aggregated amyloid beta fibrils trigger an inflammatory process that plays an important role in AD pathogenesis (Griffin and Mrak, 2002; Akiyama et al., 2000; Rojo et al., 2008). It was suggested that the inflammation occurring in the brain of AD patients has systemic parallels, and there are many reports supporting the concept that $\mathrm{AD}$ is a systemic inflammatory disease (Britschgi and WyssCoray, 2007). A number of reports also show that more T-cells are activated in AD patients than age-matched controls, and that these cells are present both in the periphery and as infiltrates in the brain (Togo et al., 2002; Town et al., 2005; Li et al., 2009). In vitro studies have shown that $A \beta$ induces the production of chemokines such as MIP$1 \alpha$, RANTES and MCP- 1 by PBMCs of AD patients and the expression of CCR5 on brain endothelial cells; this might enhance the migration of peripheral $\mathrm{T}$ cells across the blood brain barrier (BBB) ( $\mathrm{Li}$ et al., 2009; Reale et al., 2008). We have recently demonstrated that PBMC from $A D$ patients produce high levels of RANTES and MIP- $1 \beta$ after in vitro activation with $A \beta 42$, and that the expression of CCR2 and CCR5 on T cells and of CCR5 on B cells is increased in AD patients after in vitro stimulation of PBMC with $A \beta$ peptide (Pellicanò et al., 2010).

\footnotetext{
* Corresponding author. Tel.: + 49707129 81269; fax: + 497071294677

E-mail address: mariavaleria.pellicano@uni-tuebingen.de (M. Pellicanò).
}

Furthermore, evidence is accumulating for an altered distribution of lymphocyte subsets in the peripheral blood of $\mathrm{AD}$ patients compared to age-matched controls (Speciale et al., 2007). A general decrease of B and $\mathrm{T}$ cell numbers has been reported, while the number of natural killer (NK) cells was not affected (Speciale et al., 2007; Richartz-Salzburger et al., 2007). Within the T cell population, a slight increase of the percentage of CD4 + and a decrease of CD8 + lymphocytes was found (RichartzSalzburger et al., 2007). We recently reported a significant reduction of the percentage of naïve CD4 + cells (CD45RA + CCR7 + ), and an increase of effector memory (CD45RA-CCR7-) and TEMRA (CD45RA+CCR7-) cells in a pilot study of Canadian AD patients (Larbi et al., 2009) compared to age-matched controls. A reduction of $\mathrm{CD} 4+\mathrm{CD} 25^{\text {high }}$ cells, considered as potentially Treg cells, was also found (Larbi et al., 2009). No differences were discernible between $\mathrm{AD}$ patients and controls within the CD8 compartment because the effects of age were already so marked in the latter

Our previous study was limited to a small group of Canadian patients with mild $\mathrm{AD}$. To determine whether the immune signatures seen in these patients are to be expected generally in AD, we undertook a more detailed analysis on a completely different population from Italy. We have studied CD4 + and CD8 + subsets investigating the expression of the isoforms of CD45 (CD45RA and CD45RO), which can be informative for the differentiation stage of $\mathrm{T}$ cells (Michie et al., 1992; Sallusto et al., 1999), and the expression of two major positive $T$ cell costimulatory receptors CD27 and CD28 and two negative receptors expressed by late-stage differentiated cells, CD57 and KLRG1 (sometimes referred to as markers of "senescence"). CD57 is expressed on NK cells and late-stage CD8 + T cells with slight expression on CD4 + cells sometimes reported (Tarazona et al., 2000; 
Ibegbu et al., 2005), whereas KLRG1 is the Killer Lectin receptor G1, expressed on larger proportions of $\mathrm{CD} 4+\mathrm{T}$ cells as well as CD8 + $\mathrm{T}$ cells and NK cells. It was initially suggested that the expression of KLRG-1 marked replicatively-senescent cells (Voehringer et al., 2001; Voehringer et al., 2002). Finally we have analyzed the percentage of the activated $\mathrm{CD} 4+\mathrm{CD} 25+$ population that is increased in AD patients compared to young and old subjects. The emerging data suggest the existence of characteristic immune profile limited to the $\mathrm{CD} 4+\mathrm{T}$ cell subset in $\mathrm{AD}$, which might be useful diagnostically as well as in furthering understanding of the etiology of this intractable disease.

\section{Material and methods}

\subsection{Subjects}

A total of $40 \mathrm{AD}$ patients ( 22 women and 18 men; age range: $62-94$ years, mean $75 \pm 8$ ), 21 healthy old controls (11 women and 10 men; age range: $72-92$ years, mean $84 \pm 5$ ) and 11 young subjects ( 4 women and 7 men; age range: $21-28$ years, mean $25 \pm 2$ ) from Italy have been investigated. None of the controls had a history of neurodegenerative disorders. Diagnosis of probable AD was according to standard clinical procedures and followed the NINCDS/ADRDA and DSM-III-R criteria (McKhann et al., 1984; American Psychiatric Association, 1987). Cognitive performance and alterations were measured according to the mini-mental state evaluation (MMSE) and the global deterioration scale. The MMSE was normal (30/30) for the young and the healthy elderly groups. The patients were mild-tomoderate AD with MMSE scores between 25 and 10, of which 16 had mild AD (MMSE mean 22.8 \pm 2 ) and 24 moderate AD (MMSE mean $14.4 \pm 2.9$ ). All AD cases were defined as sporadic because their family history did not mention any first-degree relative with dementia. AD patients included in the study did not present with any major co-morbidity such as cancer, symptomatic (present or previous) cardiovascular diseases, or major inflammatory diseases such as autoimmunity and infections. Control subjects also had complete neurological examinations and were judged to be in good health based on their clinical history and blood tests (complete blood cell count, erythrocyte sedimentation rate, glucose, urea nitrogen, creatinine, electrolytes, C-reactive protein, liver function tests, iron, proteins, cholesterol, triglycerides). The controls were collected from the same population as the patient cohort. The University Hospital Ethics Committee approved the study, and informed consent was obtained from all guardians of patients and controls according to Italian law.

All subjects were tested for CMV serostatus by ELISA using CMVIgG-ELISA PKS assays (Genesis Diagnostics, UK). All the elderly subjects were positive for CMV antibody (both healthy old and AD) while none of the young were infected.

Whole blood was collected by venepuncture in vacutainer tubes containing ethylenediaminetetraacetic acid. Peripheral blood mononuclear cells (PBMC) were separated using a Ficoll/Hypaque gradient and frozen according to standard protocols.

\subsection{Flow cytometry}

To analyze $\mathrm{T}$ lymphocyte subsets, direct immunofluorescence was performed with anti-CD3-AlexaFluor700, CD8-PercP, CD28PercP-Cy5.5 (Becton Dickinson, Heidelberg, Germany), CD4-Qdot705, CD27-Qdot605 (Invitrogen, Karlsruhe, Germany), CD45RO-eFluor650 (eBioscience, San Diego, California), CD45RA-PacificBlue (Bio-Legend, Biozol, Eching, Germany), and CD57-FITC (Immunotools, Friesoythe, Germany). For indirect immunofluorescence, anti-human KLRG1 (clone 13A2), kindly provided by Prof. H.P. Pircher (University of Freiburg, Germany), was used as primary antibody. The secondary antibody was goat anti-mouse IgG-Qdot565 (Invitrogen). Treg analysis was performed by extracellular labeling with anti-CD3-AlexaFluor700 (Becton Dickinson), anti-CD4-Qdot705 (Invitrogen), anti-CD127-
Alexa647 (Bio-Legend) and intracellular staining with anti-FoxP3 with (Bio-Legend).

Cell viability was determined with RedVid (Invitrogen). All staining steps were performed in PFEA buffer (PBS, 2\% FCS, 2 mM EDTA and $0.01 \%$ Na Azide). Blocking of non-specific binding sites was accomplished using human immunoglobulin GAMUNEX (Bayer, Leverkusen, Germany) or mouse serum (Caltag/Invitrogen, Karlsruhe, Germany). For each experiment, cells or mouse/rat k-chain Comp Beads (Becton Dickinson) were stained with the corresponding fluorochrome-labeled antibodies and incubated for $20 \mathrm{~min}$ at $4{ }^{\circ} \mathrm{C}$ in the dark. Human unstained cells were used as negative controls. After washing with PFEA, the cells or beads were resuspended and measured using an LSR-II flow cytometer and the acquisition software FACSDiva (Becton Dickinson). Data were analyzed using Flowjo software (Tree Star, Portland, OR). For data analysis, dead cells (RedVid-positive), were excluded. CD3 + living cells were gated within the SSC/ FSC lymphocyte gate. Further analysis was performed using CD3 + CD4 + and $\mathrm{CD} 3+\mathrm{CD} 8+$ gated populations.

\subsection{Statistics}

All statistical analyses were performed with Graph-Pad Prism 4.0 using the Mann-Whitney nonparametric $U$ test to compare two independent groups. The Bonferroni correction for multiple comparisons was applied. Differences were considered significant with a $p$ value $\leq 0.005$. Significant differences are indicated by ${ }^{*} p \leq 0.005$, ${ }^{* *} \mathrm{p} \leq 0.001,{ }^{* * *} \mathrm{p} \leq 0.0005$ as mentioned in the figure legends.
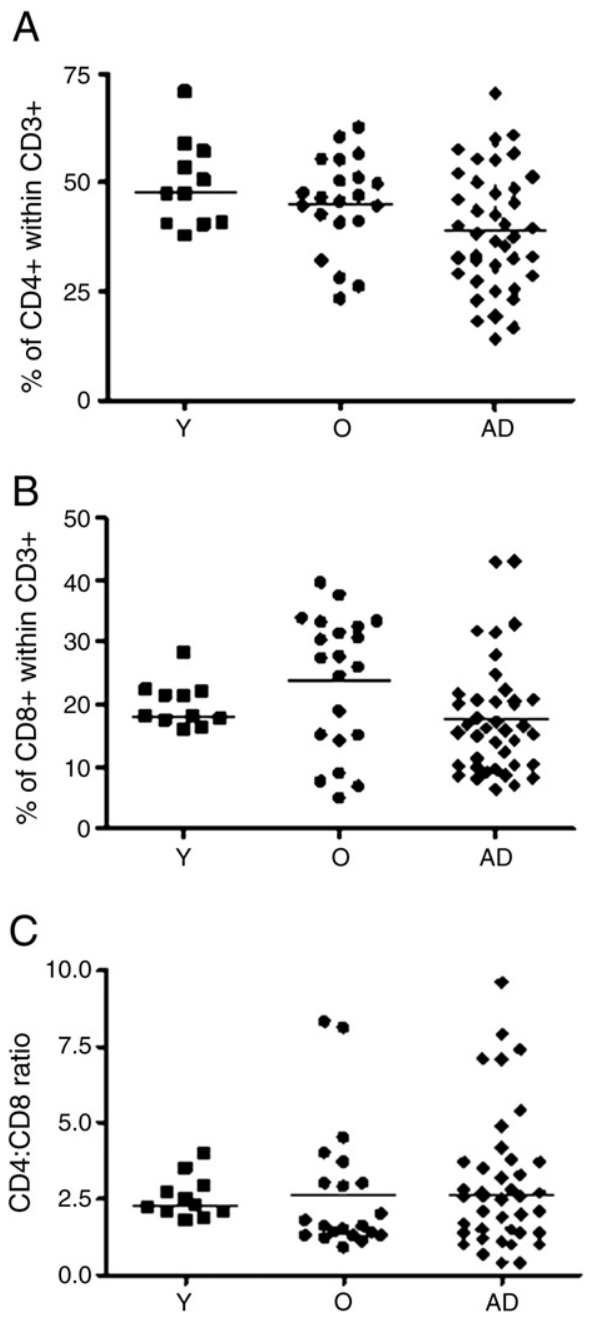

Fig. 1. Frequency of $C D 4+$ and $C D 8+$ lymphocytes in peripheral blood. Percentages of $C D 4+(A)$ and $\mathrm{CD} 8+\mathrm{T}$ lymphocytes $(\mathrm{B})$ within $\mathrm{CD} 3+$ cells, and the $\mathrm{CD} 4: \mathrm{CD} 8$ ratio $(\mathrm{C})$ in 11 young controls (squares), 21 healthy old (circles) and $40 \mathrm{AD}$ patients (diamonds). Bars represent medians. 


\section{Results}

3.1. Frequency of CD4 and CD8 cells in young controls, old controls and $A D$ patients

It has been shown that the $\mathrm{CD} 4: \mathrm{CD} 8$ ratio can be a marker predictive of mortality in an elderly Swedish population. A cluster of this marker and others constitutes the Immune Risk Profile (IRP) predicting mortality in the very elderly on 2, 4 and 6-year follow-up (Pawelec et al., 2002). Because our aim was to investigate the immune status in $\mathrm{AD}$ patients, who have a reduced lifespan compared to healthy old individuals, we have analyzed CD4 and CD8 distribution in PBMC of AD patients compared to healthy age-matched old controls and young subjects. As shown in Fig. 1A, there is a slight but non-significant decrease in the median percentage of CD4+ cells within the $\mathrm{CD} 3+\mathrm{T}$ cell population in $\mathrm{AD}$ patients as a group compared to the age-matched controls. The decrease in CD4+ cells was marginally more marked for AD patients compared to young controls, but this difference also failed to reach significance. However, within the broad range of values recorded in this group, individual $A D$ patients showed the lowest percentages of CD4 cells, lower even than the lowest age-matched controls. These tendencies were not observed in the CD8 compartment (Fig. 1B). These slight shifts also failed to affect the CD4:CD8 ratio in the three groups. However, individual patients within the AD group again showed the most extreme differences, with the lowest $\mathrm{CD} 4: 8$ ratios (Fig. $1 C$ ). The CD4:8 ratio cut-off for inclusion in the IRP is $<1$; the data summarized in Fig. 1C indicate that, as expected, no young controls fall into this group, whereas $1 / 21$ old controls (5\%), but $5 / 40 \mathrm{AD}$ patients (12.5\%) have a CD4:8 ratio of $<1$. No significant differences were observed comparing CD4:8 ratios obtained from subjects with mild vs moderate AD (data not shown).

\subsection{Costimulatory molecules on $C D 4+$ and $C D 8+$ cells}

The distribution of $\mathrm{T}$ cells at different stages of differentiation (naïve, memory etc.) is likely to be more important than the mere percentages of CD4 and CD8 cells. We therefore compared T cell differentiation status in $\mathrm{AD}$ patients versus old controls, using multiple markers including the CD27 and CD28 costimulatory molecules, which are crucial for $\mathrm{T}$ cell activation. It has been reported that expression of these two markers is reduced proportional to the degree of differentiation of the T cell (Romero et al., 2007). Naïve cells are CD28 + CD27 + while the latest stage of T cell differentiation is accompanied by the loss of both CD28 and CD27 (Koch et al., 2008). As shown in Fig. 2A (left-hand side) there is a slight but not significant decrease in the percentage of $\mathrm{CD} 28+\mathrm{CD} 27+$ cells within the CD4 + subset in AD patients compared to the elderly controls. The young controls in this population have a highly significantly greater percentage of $\mathrm{CD} 27+\mathrm{CD} 28+$ cells within the $\mathrm{CD} 4$ subset than either the old controls or AD patients, albeit more marked in the latter. Reciprocally, significantly increased percentages of late-differentiated CD4 + cells (CD28-CD27-) are seen in both elderly controls and AD patients, with no real difference between the latter two groups (Fig. 2A, right-hand side).

Within the CD8 subset, the anticipated highly significantly lower percentage of $\mathrm{CD} 27+\mathrm{CD} 28+$ and higher percentage of doublenegative cells is seen in the old compared to the young, with no difference at all between $\mathrm{AD}$ patients and age-matched controls (Fig. 2B).

\subsection{Frequencies of naïve and late-differentiated cells within the $C D 4+$ and the $C D 8+$ populations}

Although assessing the expression of CD27 and CD28 provides some information on $\mathrm{T}$ cell differentiation status and potentially $\mathrm{T}$ cell function, a combination of markers is required to distinguish between naïve and memory cells. To this end, we have analyzed the expression of CD45RA and CD45RO together with CD27 and CD28. CD45RA and CD45RO are isoforms of the protein phosphatase CD45, with naïve cells more likely to express CD45RA and memory cells CD45RO. However, re-expression of CD45RA by late-differentiated memory cells makes this marker unsuitable for identifying naïve cells by itself (Hamann et al., 1997). Thus we used four markers to identify naïve and memory subsets, as follows: CD28+CD27+
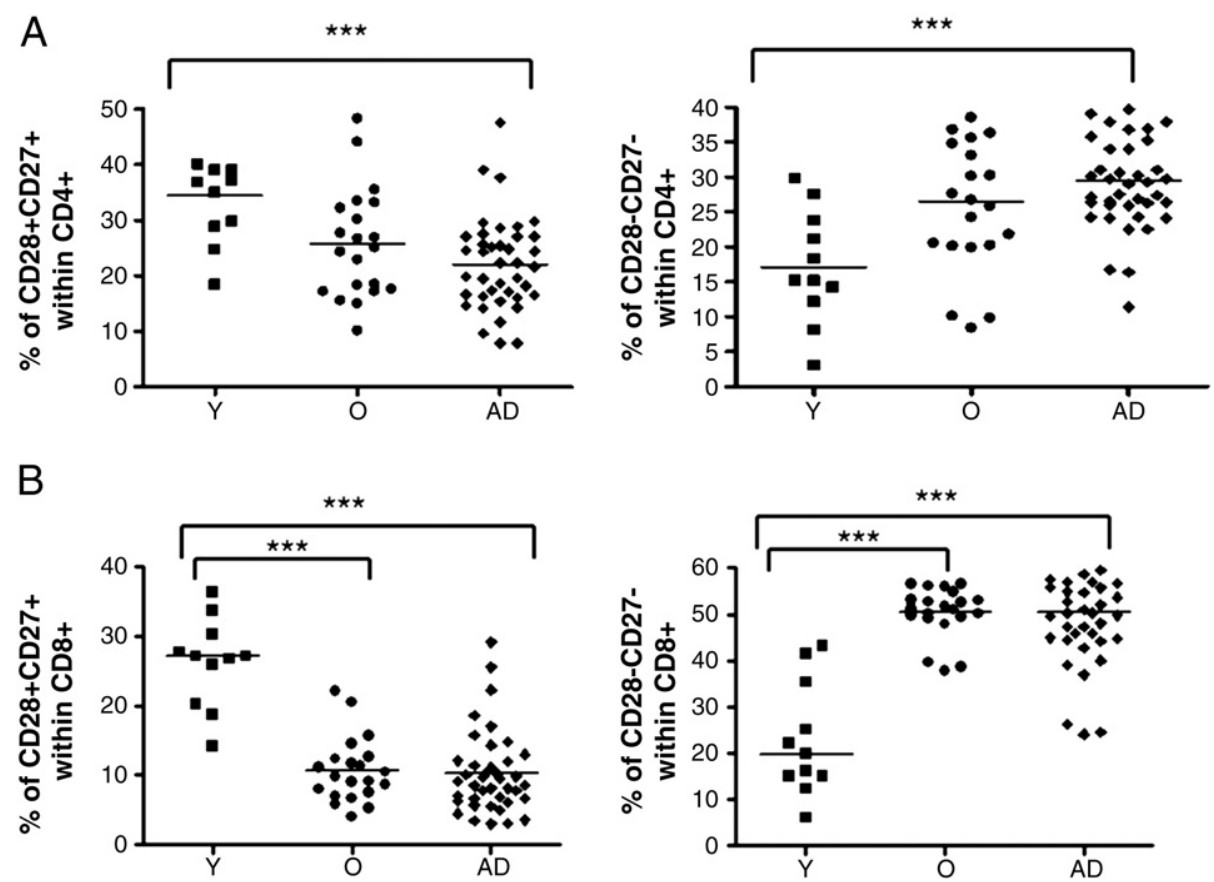

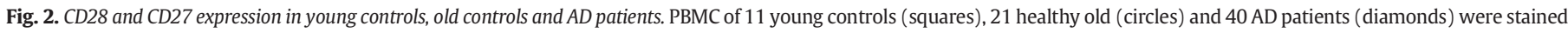

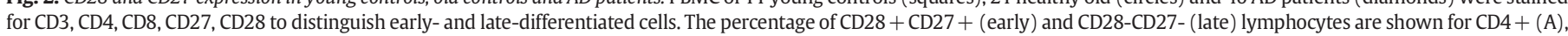
and CD8 + T cells (B). Bars represent medians. Significant differences are indicated by ${ }^{* * *} \mathrm{p} \leq 0.0005$ by Mann-Whitney nonparametric U testing with Bonferroni correction. 
CD45RA + CD45RO- presumptive naïve, and CD28-CD27-CD45RA + CD45RO + late differentiated, comparing CD4 and CD8 lymphocytes in the young, old and AD patients. Fig. 3A gives examples of flow cytometry data as dot-plots for CD4 + cells from a young donor, an old control and an AD patient, and Fig 3B shows the summed data from 11 young controls, 21 healthy elderly and the $40 \mathrm{AD}$ patients. Although the percentages of naïve CD4 cells in both old controls and $A D$ patients are very low relative to the young, the decrease in $A D$ compared to age-matched controls reaches significance. Similarly, the reciprocal increase in late-differentiated memory cells is also significantly greater in $\mathrm{AD}$ patients than in age-matched controls. These differences are not seen in the CD8 subset analyzed in the same manner (Fig. 4).

The same analysis was done comparing patients with mild vs. moderate $\mathrm{AD}$ and no significant differences have been observed (data not shown).

\subsection{Expression of "senescence" markers on $C D 4+$ and $C D 8+T$ cells}

In an effort to refine our definition of naïve and late-differentiated T cells in $\mathrm{AD}$, in order to increase our ability to distinguish immune signatures in $\mathrm{AD}$ from age-associated changes in age-matched controls, we employed two additional markers. Previous studies have shown that chronic antigenic stimulation of the immune system, such as in the case of cytomegalovirus (CMV) infection, led to increased frequencies of late- or even end-stage differentiated CD8 + T cells. These cells highly express KLRG1 and CD57 which are sometimes designed "senescence" markers (Ibegbu et al., 2005). Therefore, we tested the possibility that putative chronic stimulation of the immune system by AD-derived end products $(A \beta)$ would influence the expression of these two markers. As shown in Fig. 5, within the CD4 subset, the percentage of cells expressing KLRG-1 was very significantly greater in AD patients compared to age-matched controls (Fig. 5, upper right-hand panel). This was not the case for their CD8 + cells (Fig. 5, lower right-hand panel), and nor was it the case for either CD4 or CD8 cells regarding their expression of CD57 (Fig. 5, left-hand panels). Also in this case no significant differences have been observed comparing data obtained from patients with mild vs. moderate AD (data not shown).

\section{5. $C D 4+C D 25+$ cells and Tregs}

CD25, the IL-2 receptor $\alpha$ chain, is expressed by activated T cells, but is also taken as a marker of regulatory T cells. We have confirmed a significantly higher frequency of $\mathrm{CD} 4+\mathrm{CD} 25+\mathrm{T}$ cells in $\mathrm{AD}$ patients compared to both groups of controls. Examples of staining for CD4 and CD25 in a young control (left-hand panel), old control
A
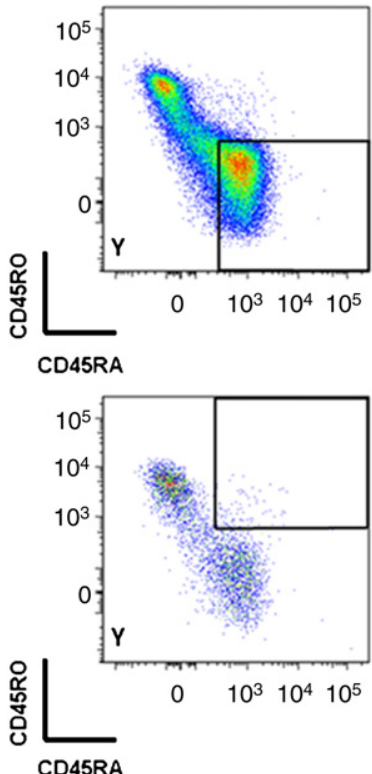

CD45RA naïve

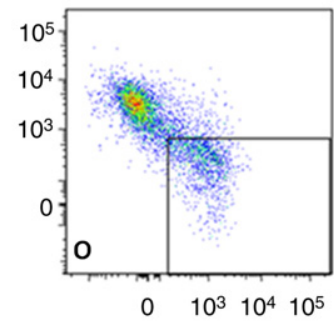

late-differentiated

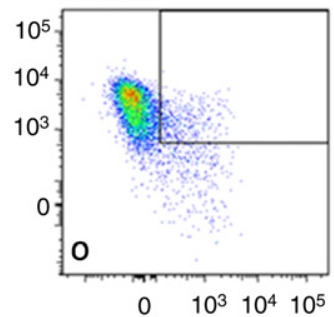

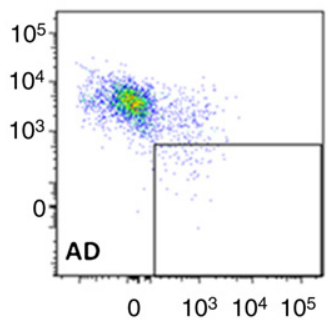

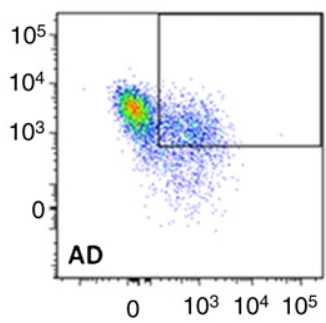

B
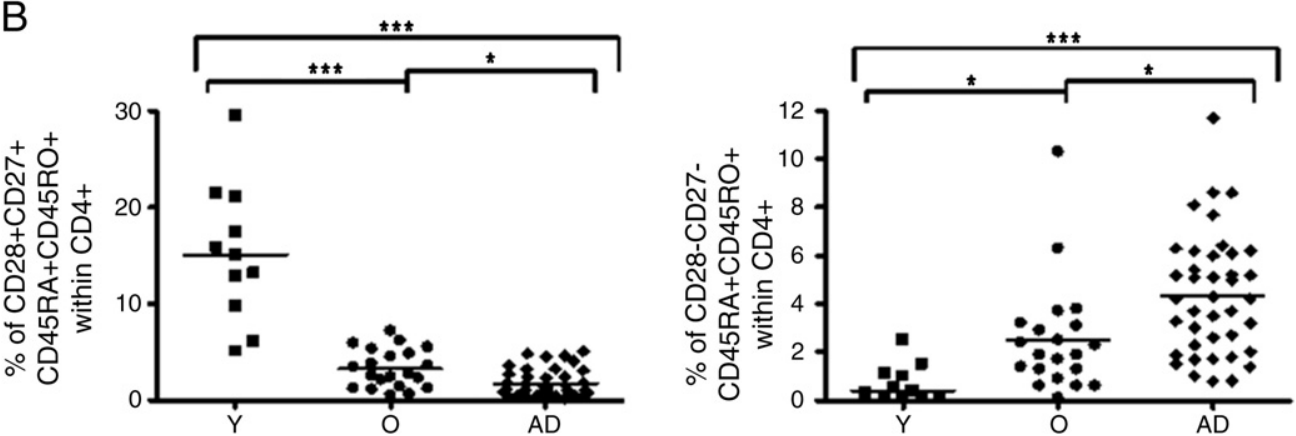

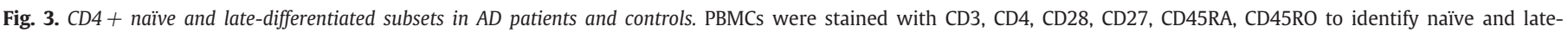

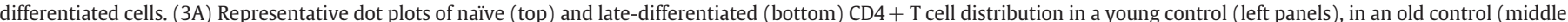

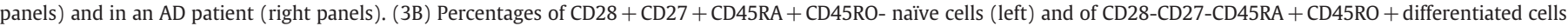

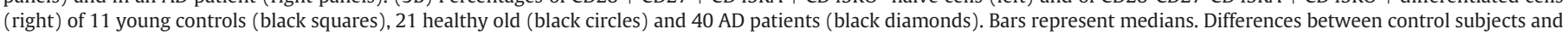
$\mathrm{AD}$ have been assessed by Mann-Whitney nonparametric $\mathrm{U}$ testing with Bonferroni correction. Significant differences are indicated by ${ }^{*} \mathrm{p} \leq 0.005$, ${ }^{* * *} \mathrm{p} \leq 0.0005$. 
A
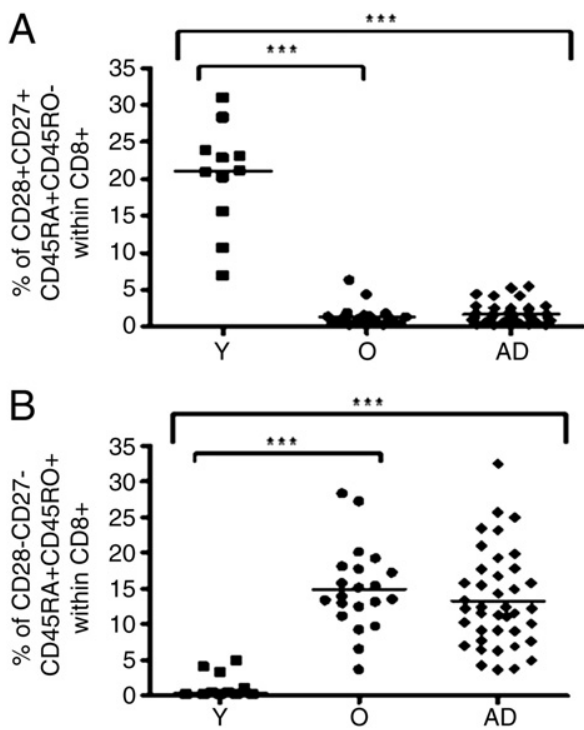

Fig. 4. $C D 8+$ naïve and late-differentiated subsets in $A D$ patients and controls. The percentages of $\mathrm{CD} 8+$ naïve $\mathrm{CD} 28+\mathrm{CD} 27+\mathrm{CD} 45 \mathrm{RA}+\mathrm{CD} 45 \mathrm{RO}$ - cells (4A) and latedifferentiated CD28-CD27-CD45RA + CD45RO + cells (4B) within PBMC of 11 young controls (black squares), 21 healthy old (black circles) and $40 \mathrm{AD}$ patients (black diamonds) are shown. The bar represents the median. Differences between control subjects and $\mathrm{AD}$ have been evaluated by Mann-Whitney nonparametric $U$ testing with Bonferroni correction. Significant differences are indicated by ${ }^{* * *} \mathrm{p} \leq 0.0005$.

(middle) and AD patient (right-hand panel) are shown in Fig. $6 \mathrm{~A}$ and the summed data from 11 young controls, 21 healthy old and $40 \mathrm{AD}$ patients in Fig. 6B, left panel. There is a highly significant difference between the old controls and the AD patients, with the latter possessing a greater percentage of CD4 + CD25 + cells. However, CD25 by itself is not sufficient to identify regulatory $\mathrm{T}$ cells; to this end we used FoxP3 nuclear staining and weak CD127 surface staining as markers for these cells. As shown in the right panel of Fig. 6B, the percentage of CD4 + CD25 + FoxP3 + CD127low T cells is higher in both elderly controls as well as AD patients compared to the young, but there is no difference between old controls and AD. This suggests that $\mathrm{AD}$ patients have increased levels of $\mathrm{CD} 4+\mathrm{T}$ cell activation but not Tregs. No significant differences were observed comparing CD24+ CD25 + obtained from subjects with mild vs moderate AD (data not shown).

\section{Discussion}

Alzheimer's disease is the most common form of dementia and represents one of the main causes of disabilities among elderly people. The diagnosis of $\mathrm{AD}$ is made following clinical criteria and only post-mortem autopsy can really confirm the disease (McKhann et al., 1984). Such clinical criteria do not allow early diagnosis of Alzheimer's disease even if the pathological alterations are present years before a certain diagnosis. The availability of reliable minimallyinvasive biomarkers for AD progression and especially for incipient AD would be vital for an early diagnosis and a timely start of appropriate treatment to slow disease progression (Schupf et al., 2008; Mocali et al., 2004; Uberti et al., 2010; Padovani et al., 2001).

The accumulation of senile plaques in the CNS formed by A $\beta$ deposits is the main hallmark of the disease. For this reason $\mathrm{AD}$ has been always considered as a brain disease (Selkoe, 2001). Recently it was suggested that the inflammation induced by the accumulation of $A \beta$ is not an only a local phenomenon but can induce systemic symptoms or be caused by systemic events (Britschgi and Wyss-Coray, 2007; Richartz-Salzburger et al., 2007; Fiala et al., 2005). Moreover, it is likely that $A B$ is not only accumulated in the brains of $A D$ patients, but is also present in the periphery and can be detected in the blood (Britschgi and Wyss-Coray, 2007; Mayeux et al., 2003; Sagare et al., 2011).

In this study we have tested the hypothesis that patients suffering from Alzheimer's disease show systemic changes at the immunological level, consistent with chronic antigenic stress potentially resulting in immune exhaustion. This question has been sporadically
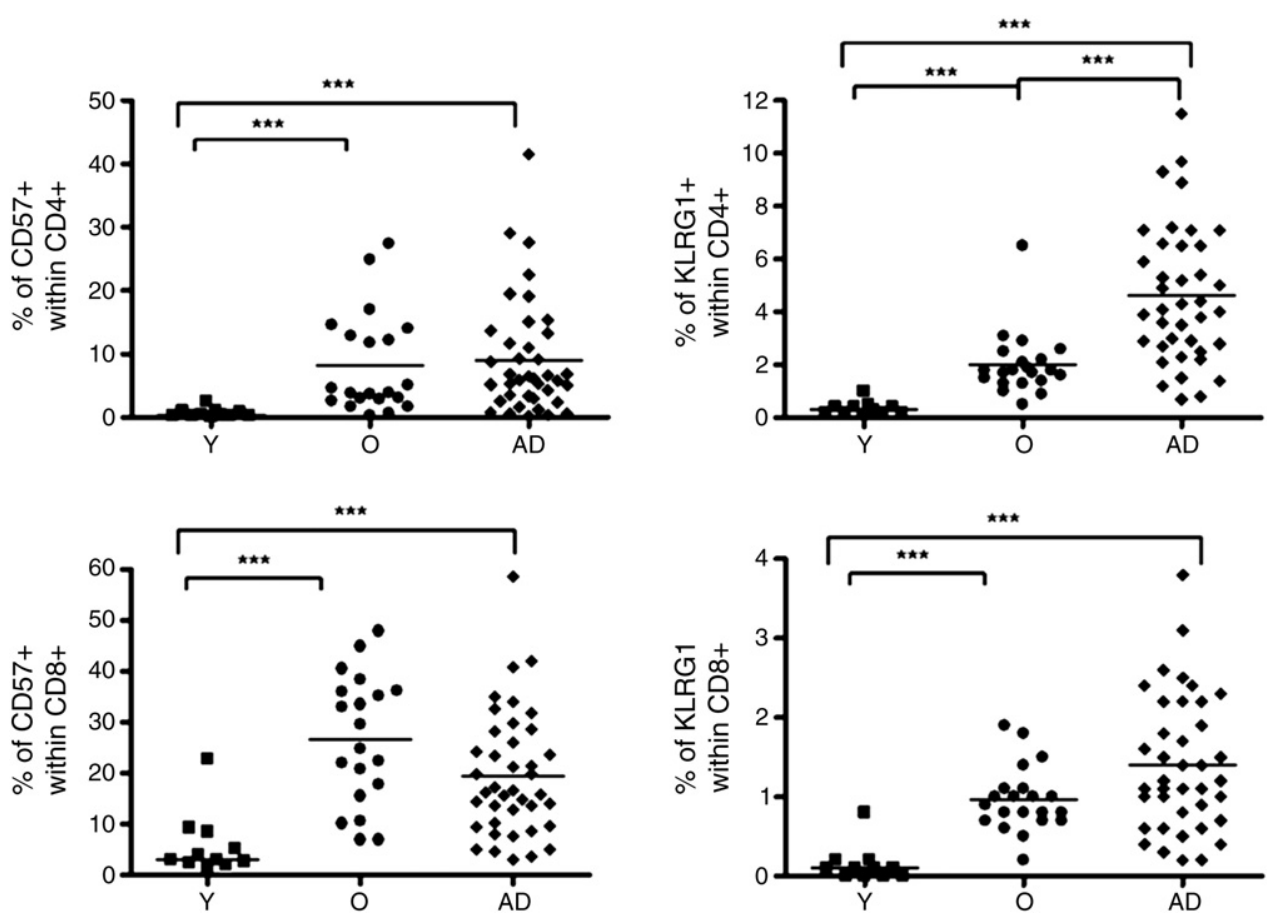

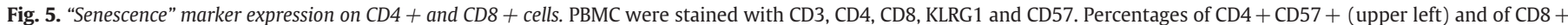

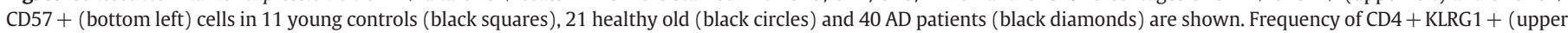

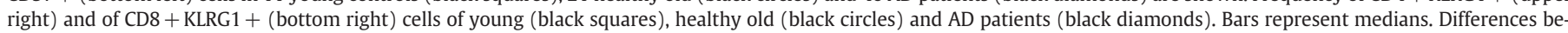

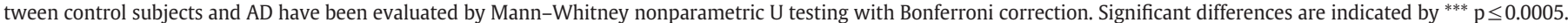


A
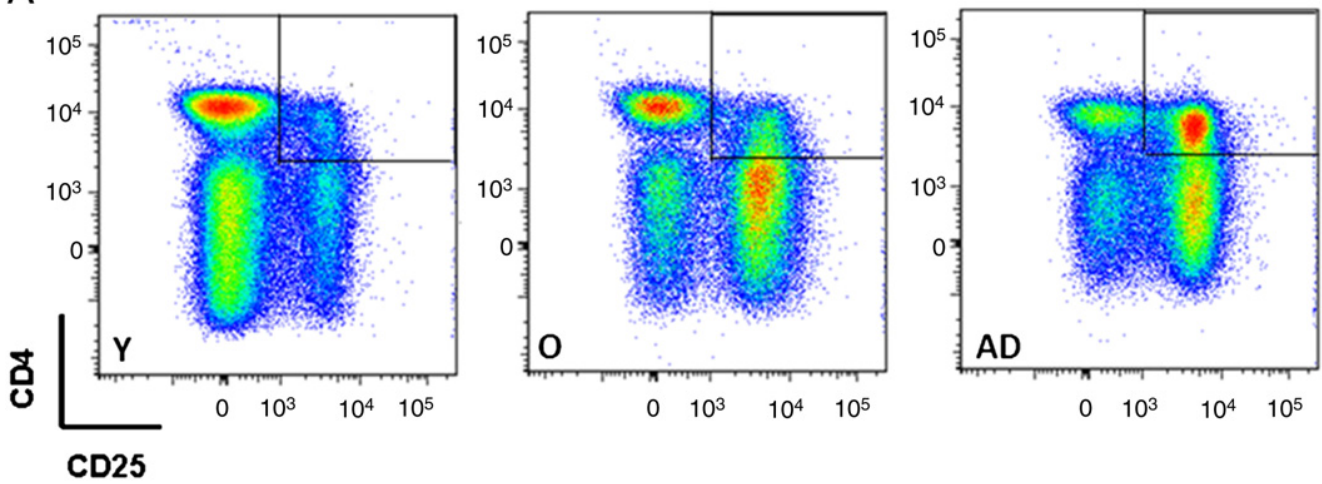

B
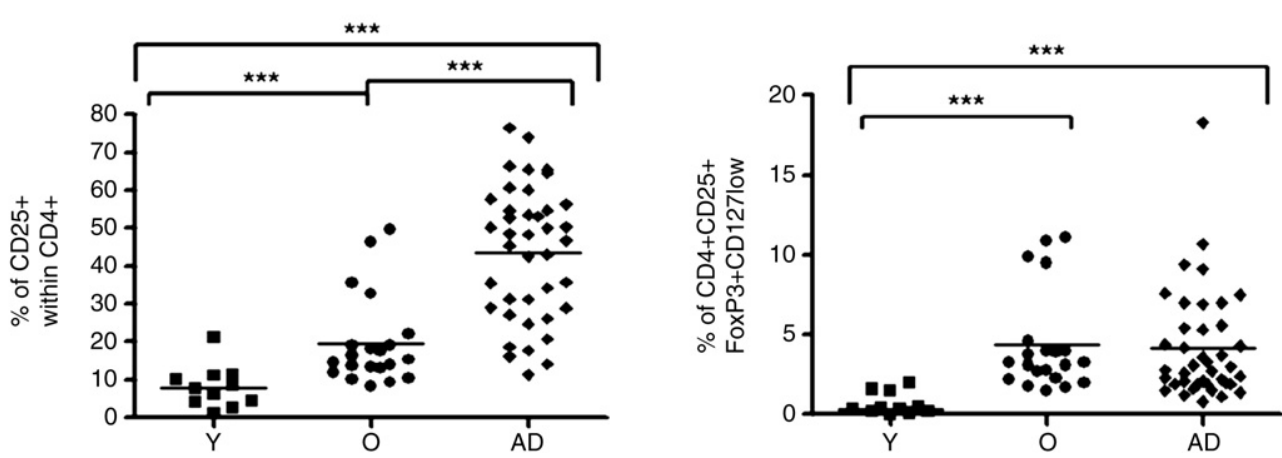

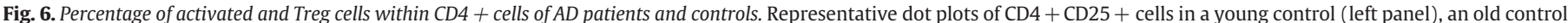

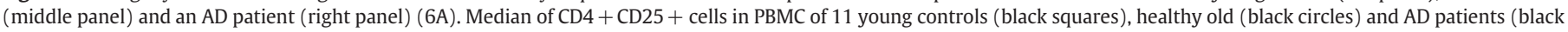

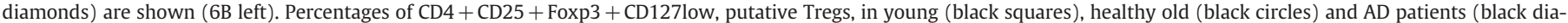

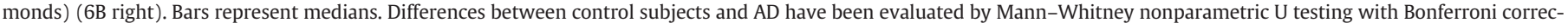
tion. Significant differences are indicated by ${ }^{* * *} \mathrm{p} \leq 0.0005$.

investigated by others in the past, with disparate results, which may have been partly the result of technical issues with analytic techniques, among other possibilities, especially regarding $\mathrm{AD}$ diagnostic criteria. A significant decrease of $\mathrm{B}$ and $\mathrm{T}$ cell percentages without changes of natural killer (NK) cells was previously reported (Richartz-Salzburger et al., 2007). These and other investigators also reported a slight increase in the percentage of CD4 + T cells and a decrease of CD8 + cells (Richartz-Salzburger et al., 2007; Lombardi et al., 1999). These data are not in complete agreement with other reports of no differences in the percentage and absolute number of CD3 $+\mathrm{T}$ cells, NK cells or CD4 + and CD8 + T cells (Speciale et al., 2007). In our own pilot study we showed that major changes are seen within the $\mathrm{CD} 4+\mathrm{T}$ cell subset in mild AD patients compared to healthy elderly controls (Larbi et al., 2009) whereas the more marked changes in the CD8 subsets were seen equally in both patients and age-matched controls. To confirm and extend our previous findings in a different population (Italian not Canadian), here we tested a larger group of AD patients $(n=40$, one group of mild and one group of moderate AD) compared to healthy old $(n=21)$ and young subjects $(n=11)$ using multiparameter flow cytometry, including new markers to better characterize the immunological profile of these patients.

The analysis of costimulatory molecules revealed a slight reduction of CD28 + CD27 + (early differentiated cells) and an increased percentage of CD28-CD27- (late differentiated cells) within the CD4+ subset of both mild and moderate AD patents compared to healthy elderly controls. We extended this analysis to include CD45 isoform expression, and found a significantly reduced median percentage of CD28 + CD27 + CD45RA + CD45RO- naïve CD4 + T cells in AD patients compared to old controls and a reciprocal increase of CD4+ CD28-CD27-CD45RA + CD45RO + late-differentiated memory cells. Furthermore, we tested the expression of the potential senescence markers CD57 and KLRG1 on these cell subpopulations. The results with KLRG-1 revealed the most marked differences found here between $\mathrm{AD}$ and age-matched controls, with highly significantly greater percentages of CD4 + T cells carrying this marker in the former. The CD57 marker was not informative in this respect. Neither were any of these markers informative within the $\mathrm{CD} 8$ subset, probably because the age-associated changes already seen in the controls were so marked that any additional alterations in the patient group were not visible. We know that the age-associated changes seen in $\mathrm{CD} 8+\mathrm{T}$ cells are predominantly caused by persistent infection with, and hence chronic antigenic stimulation by, CMV, because CMV-negative individuals do not manifest these changes at older age (Chidrawar et al., 2009; Derhovanessian et al., 2010). In contrast, the CD4 + cells are only marginally or much less affected by CMV serostatus (Derhovanessian et al., 2010). Thus, the striking findings of differences within the $\mathrm{CD} 4$ subset between $\mathrm{AD}$ patients and age-matched controls reported here cannot be caused by CMV; also all elderly controls as well as AD patients were CMV-seropositive. Because all young controls were CMV-seronegative, the even more marked differences between young and old groups are likely to be predominantly a result of CMV stratification. It was discussed by Goronzy et al. that there is an effective homeostatic control mechanism that allows the maintenance of an appropriate number of naïve CD4 $+\mathrm{T}$ cells in elderly people, in contrast with the CD8 + compartment that is more shifting with a decrease of naïve CD8 + cells already during middle age (Goronzy et al., 2007). Only in some pathological conditions with inflammatory components (e.g. rheumatoid arthritis) it was shown that chronic antigenic stress can be responsible for premature immunosenescence of CD4 + cells (Weyand and Goronzy, 2002). We hypothesize that the differences in CD4 + naïve, memory and late differentiated (KLRG1 +) $\mathrm{T}$ cell distribution between $\mathrm{AD}$ and age-matched controls are causes by chronic antigenic stimulation by $A \beta$ present in the blood but this has not been directly tested and a differential effect of CMV in AD- 
vs-age matched controls cannot be ruled out. Alternatively the shift from naïve to late memory $C D 4+$ cells in $A D$ patients can be the result of the capture of $A \beta$ by local APC in the brain and the migration of these cells towards secondary lymph nodes inducing $T$ cell stimulation, as suggested by Monsonego et al. (Monsonego et al., 2003). The present results are consistent with our previous report of a pilot study of mild AD patients (Larbi et al., 2009), but extend that study beyond the diagnosis of mild AD to include moderate AD. Moreover, the employment of a different set of markers to define naïve and differentiated memory cells in these two studies in patients from two different countries, nonetheless with consistent results, suggests that these findings are likely to be robust. Furthermore, it may be of consequence that the immune profile seen here in moderate $A D$ is already present in the mild $A D$ group. This suggests that immune changes occur early in disease progression and raises the possibility that they may occur even before cognitive symptoms manifest. If this were so, it has implications for early diagnosis of alterations leading to the future development of $\mathrm{AD}$. We are currently testing this possibility in patients with mild cognitive or no impairment.

In our previous paper we also showed a higher percentage of CD4+ CD25 + putative Treg cells in AD compared to healthy controls (Larbi et al., 2009). However, the IL $2 \mathrm{r} \alpha$ chain (CD25) expressed on Tregs is also expressed on activated cells. To better identify these cells we used several other Treg markers, and found a markedly higher frequency only of CD4 + CD25 + FoxP3- cells (i.e. activated T cells, not Tregs) in $\mathrm{AD}$ patients compared to old controls. These data are in agreement with others reporting a higher frequency of activated cells in the PBMC of AD patients (Pellicanò et al., 2010; Monsonego et al., 2003; Miscia et al., 2009; Ciccocioppo et al., 2008), but seem discordant with a recent report on increased levels of Tregs in AD (Saresella et al., 2010). The presence of activated $C D 4+T$ cells might be the result of $A \beta$-specific chronic $\mathrm{T}$ cell stimulation, creating a pro-inflammatory environment, and enhancing disease progression. Possibly, the level of Treg induction depends on disease progression, because Sarasella et al. found greater suppressive activity in mild cognitively impaired patients than in AD patients (Saresella et al., 2010).

We conclude that there is a common peripheral immune profile for Alzheimer's disease which mainly involves CD4 $+\mathrm{T}$ cells, changes to which are consistent with chronic antigenic stress leading to immune exhaustion. Whether $A \beta$ is really the driving force is unknown but efforts will be concentrated on this issue in order to define how and why CD4+ $\mathrm{T}$ cells undergo changes in $\mathrm{AD}$. It will be crucial to determine whether such changes to $C D 4 T$ cells precede the development of $A D$, whether they are discernible in $\mathrm{MCI}$, or even in people not yet showing any cognitive impairment. To this end, we are going to study the immune profile of different forms of dementia to see whether the differences we observed for CD4 + cells are specific to AD and can be used as biomarkers for early diagnosis. Moreover, focusing intervention efforts on CD4 cells to alleviate their potential dysregulation could be of therapeutic benefit. This could possibly be approached by targeting CCR5.

\section{Acknowledgments}

We would like to thank Prof. Hans-Peter Pircher for providing the anti-KLRG1 antibody, and Lilly Oettinger for antibody titration and flow cytometry quality controls. This study was supported by EU project LifeSpan (FP6 036894) and by DFG-PA 361/14-1. DG is a member of the DFG Graduate School GRK794.

\section{References}

Akiyama, H., Barger, S., Barnum, S., Bradt, B., Bauer, J., Cole, G.M., Cooper, N.R., Eikelenboom, P., Emmerling, M., Fiebich, B.L., Finch, C.E., Frautschy, S., Griffin, W.S., Hampel, H., Hull, M., Landreth, G., Lue, L., Mrak, R., Mackenzie, I.R., McGeer, P.L., O'Banion, M.K., Pachter J., Pasinetti, G., Plata-Salaman, C., Rogers, J., Rydel, R., Shen, Y., Streit, W., Strohmeyer, R., Tooyoma, I., Van Muiswinkel, F.L., Veerhuis, R., Walker, D., Webster, S., Wegrzyniak, B., Wenk, G., Wyss-Coray, T., 2000. Inflammation in Alzheimer's disease. Neuroinflammation working group. Neurobiol. Aging 21, 383-421.
American Psychiatric Association Diagnostic and Statistical Manual of Mental Disorders: DSM-III-R, 3rd revised ed. American Psychiatric Association, Washington (DC).

Britschgi, M., Wyss-Coray, T., 2007. Systemic and acquired immune responses in Alzheimer's disease. Int. Rev. Neurobiol. 82, 205-233.

Chidrawar, S., Khan, N., Wei, W., McLarnon, A., Smith, N., Nayak, L., Moss, P., 2009. Cytomegalovirus-seropositivity has a profound influence on the magnitude of major lymphoid subsets within healthy individuals. Clin. Exp. Immunol. 155 423-432.

Ciccocioppo, F., Lanuti, P., Marchisio, M., Gambi, F., Santavenere, E., Pierdomenico, L., Bascelli, A., Velluto, L., Gambi, D., Miscia, S., 2008. Expression and phosphorylation of protein kinase $C$ isoforms in Abeta(1-42) activated T lymphocytes from Alzheimers disease. Int. J. Immunopathol. Pharmacol. 21, 23-33.

Derhovanessian, E., Maier, A.B., Beck, R., Jahn, G., Hahnel, K., Slagboom, P.E., de Craen, A.J., Westendorp, R.G., Pawelec, G., 2010. Hallmark features of immunosenescence are absent in familial longevity. J. Immunol. 185, 4618-4624.

Fiala, M., Lin, J., Ringman, J., Kermani-Arab, V., Tsao, G., Patel, A., Lossinsky, A.S., Graves, M.C., Gustavson, A., Sayre, J., Sofroni, E., Suarez, T., Chiappelli, F., Bernard, G., 2005. Ineffective phagocytosis of amyloid-beta by macrophages of Alzheimer's disease patients. J. Alzheimers Dis. 7, 221-232.

Goronzy, J.J., Lee, W.W., Weyand, C.M., 2007. Aging and T-cell diversity. Exp. Gerontol. 42 (5), 400-406.

Griffin, W.S., Mrak, R.E., 2002. Interleukin-1 in the genesis and progression of and risk for development of neuronal degeneration in Alzheimer's disease. J. Leukoc. Biol. 72 (2), 233-238.

Hamann, D., Baars, P.A., Rep, M.H., Hooibrink, B., Kerkhof-Garde, S.R., Klein, M.R., van Lier, R.A., 1997. Phenotypic and functional separation of memory and effector human CD8 + T cells. J. Exp. Med. 186, 1407-1418.

Ibegbu, C.C., Xu, Y.X., Harris, W., Maggio, D., Miller, J.D., Kourtis, A.P., 2005. Expression of killer cell lectin-like receptor G1 on antigen-specific human CD8 + T lymphocytes during active, latent, and resolved infection and its relation with CD57. J. Immunol. 174 (10), 6088-6094.

Koch, S., Larbi, A., Derhovanessian, E., Özcelik, D., Naumova, E., Pawelec, G., 2008. Multiparameter flow cytometric analysis of CD4 and CD8 T cellsubsets in young and old people. Immun. Ageing 5, 6 .

Larbi, A., Pawelec, G., Witkowski, J.M., Schipper, H.M., Derhovanessian, E., Goldeck, D., Fulop, T., 2009. Dramatic shifts in circulating CD4 but not CD8 T cell subsets in mild Alzheimer's disease. J. Alzheimers Dis. 17 (1), 91-103.

Li, M., Shang, D.S., Zhao, W.D., Tian, L., Li, B., Fang, W.G., Zhu, L., Man, S.M., Chen, Y.H., 2009. Amyloid beta interaction with receptor for advanced glycation end products up-regulates brain endothelial CCR5 expression and promotes T cells crossing the blood-brain barrier. J. Immunol. 182 (9), 5778-5788.

Lombardi, V.R., Garcia, M., Rey, L., Cacabelos, R., 1999. Characterization of cytokine production, screening of lymphocyte subset patterns and in vitro apoptosis in healthy and Alzheimer's Disease (AD) individuals. J. Neuroimmunol. 97, 163-171.

Mayeux, R., Honig, L.S., Tang, M.X., Manly, J., Stern, Y., Schupf, N., Mehta, P.D., 2003 PlasmaA[beta]40 and A[beta]42 and Alzheimer's disease: relation to age, mortality, and risk. Neurology 61, 1185-1190.

McKhann, G., Drachman, D., Folstein, M., Katzman, R., Price, D., Stadlan, E.M., 1984 Clinical diagnosis of Alzheimer's disease: report of the NINCDS-ADRDA Work Group under the auspices of Department of Health and Human Service Task Force on Alzheimer's Disease. Neurology 34, 939-944.

Michie, C.A., McLean, A., Alcock, C., Beverley, P.C., 1992. Lifespan of human lymphocyte subsets defined by CD45 isoforms. Nature 360, 264-265.

Miscia, S., Ciccocioppo, F., Lanuti, P., Velluto, L., Bascelli, A., Pierdomenico, L., Genovesi, D., Di Siena, A., Santavenere, E., Gambi, F., Ausili-Cefaro, G., Grimley, P.M., Marchisio M., Gambi, D., 2009. Abeta(1-42) stimulated T cells express P-PKC-delta and P-PKCzeta in Alzheimer disease. Neurobiol. Aging 30, 394-406.

Mocali, A., Cedrola, S., Della Malva, N., Bontempelli, M., Mitidieri, V.A., Bavazzano, A. Comolli, R., Paoletti, F., La Porta, C.A., 2004. Increased plasma levels of soluble CD40, together with the decrease of TGF beta 1 , as possible differential markers of Alzheimer disease. Exp. Gerontol. 39 (10), 1555-1561.

Monsonego, A., Zota, V., Karni, A., Krieger, J.I., Bar-Or, A., Bitan, G., Budson, A.E., Sperling, R., Selkoe, D.J., Weiner, H.L., 2003. Increased T cell reactivity to amyloid beta protein in older humans and patients with Alzheimer disease. J. Clin. Invest. 112, 415-422.

Padovani, A., Pastorino, L., Borroni, B., Colciaghi, F., Rozzini, L., Monastero, R., Perez, J., Pettenati, C., Mussi, M., Parrinello, G., Cottini, E., Lenzi, G.L., Trabucchi, M., Cattabeni, F., Di Luca, M., 2001. Amyloid precursor protein in platelets: a peripheral marker for the diagnosis of sporadic AD. Neurology 57 (12), 2243-2248.

Pawelec, G., Ouyang, Q., Colonna-Romano, G., Candore, G., Lio, D., Caruso, C., 2002. Is human immunosenescence clinically relevant? Looking for 'immunological risk phenotypes'. Trends Immunol. 23, 330-332.

Pellicanò, M., Bulati, M., Buffa, S., Barbagallo, M., Di Prima, A., Misiano, G., Picone, P., Di Carlo, M., Nuzzo, D., Candore, G., Vasto, S., Lio, D., Caruso, C., Colonna-Romano, G., 2010. Systemic immune responses in Alzheimer's disease: in vitro mononuclear cell activation and cytokine production. J. Alzheimers Dis. 21 (1), 181-192.

Reale, M., Iarlori, C., Feliciani, C., Gambi, D., 2008. Peripheral chemokine receptors, their ligands, cytokines and Alzheimer's disease. J. Alzheimers Dis. 14 (2), 147-159.

Richartz-Salzburger, E., Batra, A., Stransky, E., Laske, C., Köhler, N., Bartels, M., Buchkremer, G., Schott, K., 2007. Altered lymphocyte distribution in Alzheimer's disease. J. Psychiatr. Res. 41 (1-2), 174-178.

Rojo, L.E., Fernández, J.A., Maccioni, A.A., Jimenez, J.M., Maccioni, R.B., 2008. Neuroinflammation: implications for the pathogenesis and molecular diagnosis of Alzheimer's disease. Arch. Med. Res. 39 (1), 1-16.

Romero, P., Zippelius, A., Kurth, I., Pittet, M.J., Touvrey, C., Iancu, E.M., Corthesy, P., Devevre, E., Speiser, D.E., Rufer, N., 2007. Four functionally distinct populations of human effector-memory CD8 + T lymphocytes. J. Immunol. 178, 4112-4119. 
Sagare, A.P., Deane, R., Zetterberg, H., Wallin, A., Blennow, K., Zlokovic, B.V., 2011. Impaired lipoprotein receptor-mediated peripheral binding of plasma amyloid-beta is an early biomarker for mild cognitive impairment preceding Alzheimer's disease. J. Alzheimers Dis. 24 (1), 25-34.

Sallusto, F., Lenig, D., Förster, R., Lipp, M., Lanzavecchia, A., 1999. Two subsets of memory $\mathrm{T}$ lymphocytes with distinct homing potentials and effector functions. Nature 401 (6754), 708-712.

Saresella, M., Calabrese, E., Marventano, I., Piancone, F., Gatti, A., Calvo, M.G., Nemni, R Clerici, M., 2010. PD1 negative and PD1 positive CD4+T regulatory cells in mild cognitive impairment and Alzheimer's disease. J. Alzheimers Dis. 21, 927-938.

Schupf, N., Tang, M.X., Fukuyama, H., Manly, J., Andrews, H., Mehta, P., Ravetch, J. Mayeux, R., 2008. Peripheral Abeta subspecies as risk biomarkers of Alzheimer's disease. Proc. Natl. Acad. Sci. U. S. A. 105 (37), 14052-14057.

Selkoe, D.J., 2001. Alzheimer's disease results from the cerebral accumulation and cytotoxicity of amyloid beta-protein. J. Alzheimers Dis. 3 (1), 75-80.

Speciale, L., Calabrese, E., Saresella, M., Tinelli, C., Mariani, C., Sanvito, L., Longhi, R. Ferrante, P., 2007. Lymphocyte subset patterns and cytokine production in Alzheimer's disease patients. Neurobiol. Aging 28, 1163-1169.

Tarazona, R., DelaRosa, O., Alonso, C., Ostos, B., Espejo, J., Peña, J., Solana, R., 2000. Increased expression of NK cell markers on T lymphocytes in aging and chronic activation of the immune system reflects the accumulation of effector/senescent T cells. Mech. Ageing Dev. 121 (1-3), 77-88.

Togo, T., Akiyama, H., Iseki, E., Kondo, H., Ikeda, K., Kato, M., Oda, T., Tsuchiya, K., Kosaka, K., 2002. Occurrence of T cells in the brain of Alzheimer's disease and other neurological diseases. J. Neuroimmunol. 124 (1-2), 83-92.

Town, T., Tan, J., Flavell, R.A., Mullan, M., 2005. T-cells in Alzheimer's disease. Neuromolecular Med. 7 (3), 255-264 Review..

Uberti, D., Cenini, G., Bonini, S.A., Barcikowska, M., Styczynska, M., Szybinska, A., Memo, M., 2010. Increased CD44 gene expression in lymphocytes derived from Alzheimer disease patients. Neurodegener. Dis. 7 (1-3), 143-147.

Voehringer, D., Blaser, C., Brawand, P., Raulet, D.H., Hanke, T., Pircher, H., 2001. Viral infections induce abundant numbers of senescent CD8 T cells. J. Immunol. 167 (9), 4838-4843.

Voehringer, D., Koschella, M., Pircher, H., 2002. Lack of proliferative capacity of human effector and memory T cells expressing killer cell lectinlike receptor G1 (KLRG1). Blood 100 (10), 3698-3702.

Weyand, C.M., Goronzy, J.J., 2002. Premature immunosenescence in rheumatoid arthritis. J. Rheumatol. 29 (6), 1141-1146. 